\title{
Managing patients with deliberate self harm admitted to an accident and emergency observation ward
}

\author{
J Ryan, S Clemmett, C Perez-Avila
}

\begin{abstract}
Objective-To review the case records of patients admitted to an accident and emergency (A\&E) observation ward following deliberate self harm.

Methods-The hospital notes of 568 patients admitted during one year following episodes of deliberate self harm were reviewed. The study was retrospective. Results-The majority of these patients had taken an overdose and were between 18 and 35 years of age. Most patients were admitted to the observation ward after midnight or in the evening and were subsequently managed by an A\&E based deliberate self harm team. Only $20 \%$ of admissions required evaluation by a psychiatrist. Most patients were discharged the next day without further follow up.

Conclusions-The use of a specialised A\&E based team and an A\&E observation ward is appropriate for the management of many deliberate self harm patients.

( $\mathcal{A}$ Accid Emerg Med 1996;13:31-33)
\end{abstract}

Key terms: deliberate self-harm; accident and emergency observation ward.

In recent years patients with psychiatric problems have placed an increased workload on accident and emergency ( $A \& E$ ) departments. The recent shift towards care in the community for mental health patients has resulted in many psychiatric patients presenting to $A \& E$ departments during a crisis. In the past, recommendations for managing patients with attempted suicide suggested that all such patients should be reviewed by a psychiatrist. $^{1}$ More recent reports have accepted that not all patients who intentionally harm themselves require psychiatric evaluation. ${ }^{2}{ }^{3}$ In our A\&E department we have developed a protocol for managing patients with $\mathrm{DSH}$. A number of patients are subsequently admitted to the $A \& E$ observation ward where they are managed by a specialised $A \& E$ based deliberate self harm (DSH) team.

\section{Methods}

Patients who attend the A\&E department with deliberate self harm are immediately triaged as high priority patients by experienced nursing staff. The A\&E doctor performs a physical examination and appropriate treatment is given. A psychosocial assessment is made to identify those patients in a high suicide risk group. Patients are assessed using the modified SAD PERSON'S score (appendix). This assesses 10 significant risk factors for suicidal patients. Each risk factor, if present, scores one point. It is calculated on each patient and is used as an objective tool to assess the risk of suicide. A score of $<3$ is taken as low risk, 3-6 as medium risk, and $>6$ as high risk.

Medium and high risk patients are admitted to the $A \& E$ observation ward under the care of the on-call $A \& E$ consultant for an evaluation by the specialised DSH team, provided there are no significant complications requiring medical or surgical intervention. The $A \& E$ based DSH team is composed of an A\&E consultant or senior registrar, ward sister, social worker, psychiatric liaison nurse, physiotherapist, and community liaison sister.

Patients with deliberate self harm seen in the A\&E department may be discharged after appropriate intervention. Some take their own discharge or do not wait to be seen. A small number are seen in the $A \& E$ department by a psychiatrist and admitted to a psychiatric unit. Some patients are admitted under the care of the on-call medical, surgical, or orthopaedic team for more specialisted treatment.

We reviewed the notes of all patients who presented to the A\&E department during 1993 with an episode of deliberate self harm. In particular we examined the details of patients who were admitted overnight to the observation ward.

\section{Results}

A total of 1736 patients with deliberate self harm presented to the $\mathrm{A} \& \mathrm{E}$ department of the Royal Sussex County Hospital during the year 1993 , accounting for $3.2 \%$ of attendances. The department sees in excess of 54000 new patients per year. Of these, 803 were seen by A\&E staff and were discharged following appropriate management, as mandated by the departmental protocol. Three hundred and seventeen patients were admitted under the care of the duty physicians or surgeons for management of more serious complaints such as toxic overdoses or self inflicted injury requiring surgery. Five hundred and eighty six patients were admitted under the care of the $\mathrm{A} \& \mathrm{E}$ team to the A\&E observation ward.

The majority of patients admitted to the observation ward (95\%) had taken an overdose as the method of deliberate self harm (fig 1).

No significant seasonal variation was found in the number of admissions. April had the fewest admissions while November and 


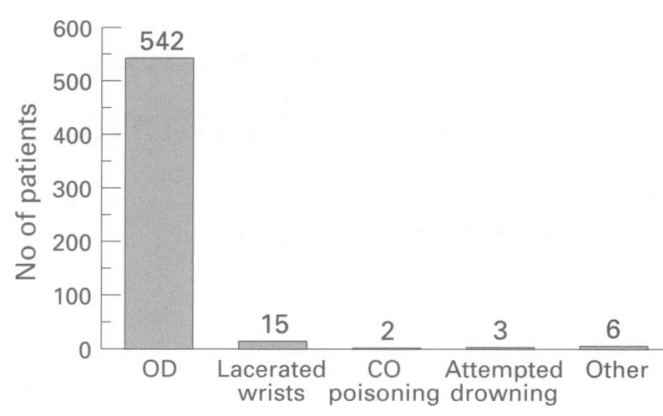

Fig 1 Method of deliberate self harm. "Other" includes: punched wall (1), lacerations to body (2), jumped under train (1), swallowed razor blade (1), intentional road traffic accident (1).

February had the highest numbers. The majority of patients were admitted to the observation ward after midnight $(49 \%) ; 39 \%$ were admitted between 16:00 hours and midnight; $12 \%$ were admitted during normal working hours.

Patients were seen most frequently by the specialised team on Sundays $(16 \%)$. Saturday $(12 \%)$ was the least busy day for the team. There was little variation in the workload for other days.

There was a slight gender bias in favour of females $(53 \%)$.

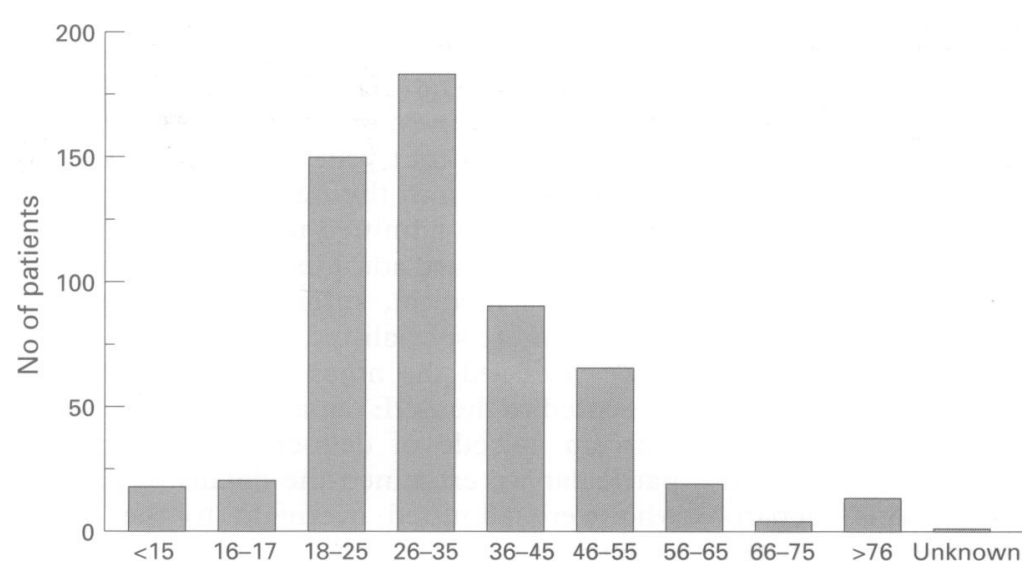

Fig 2 Age variation in patients with deliberate self harm.

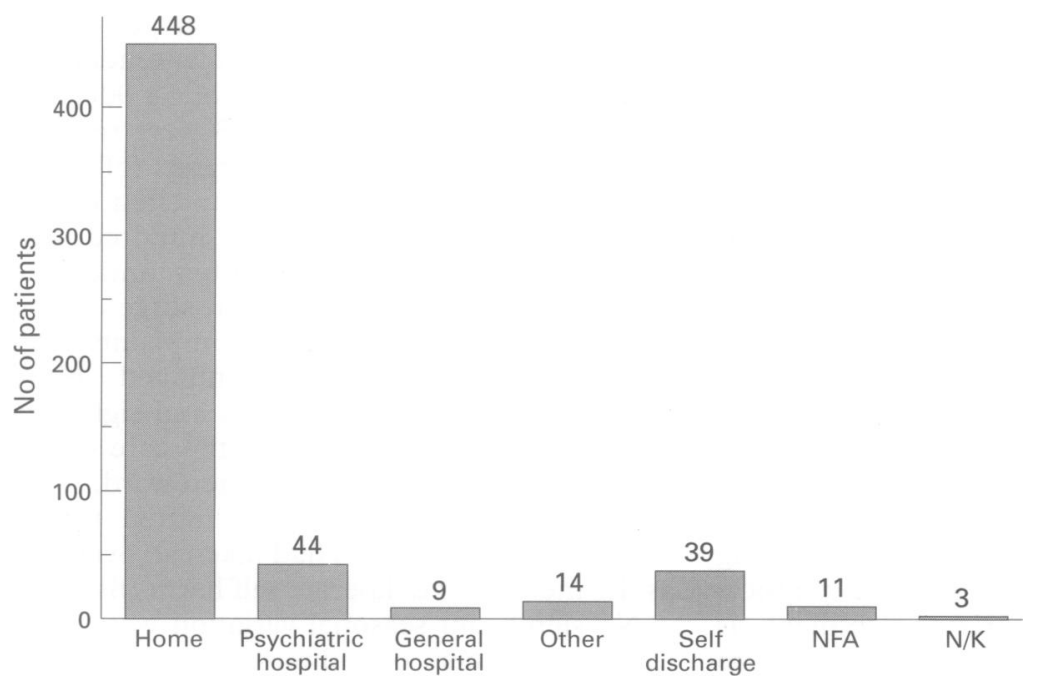

Fig 3 Disposal of patients with deliberate self harm.
Sixty six per cent of cases were under the age of 35 years. Peak age for admissions was in the 26 to 35 year age group, which contained $32.6 \%$ of cases. This was constant for all months (fig 2).

Most cases were not referred to any psy- m chiatric service. Twenty per cent were referred to the duty psychiatrist before discharge, while $10 \%$ were referred to other psychiatric services 3 such as a community psychiatric nurse, or were given a psychiatric outpatient appointment. $\mathrm{A} \stackrel{\Rightarrow}{\vec{\Rightarrow}}$ few took their own discharge before proper assessment or referral could be made. Most patients were discharged home from the observation ward (79\%) (fig 3 ).

\section{Discussion}

Patients with deliberate self harm contribute significantly to the work of the modern A\&E department. Attendances with deliberate self harm at our $A \& E$ department are almost double the number reported in other studies. These patients occupy a disproportionate amount of nursing and medical time because of their multiple psychiatric and social prob- $\omega$ lems and because of their frequent abuse of alcohol. The frequency with which they present outside of normal working hours has implications for the staffing levels of $A \& E$ departments.

We agree with recently recommended standards in the management of patients with deliberate self harm. All new members of medical staff receive training during their first week in the department in the assessment and management of the DSH patient.

In many hospitals, DSH patients are assessed by the duty psychiatrist on the day of presentation. In others, these patients are admitted under the care of the physicians and a psychiatric evaluation is requested either on the day of admission or on the following day. One study reports $61 \%$ of admissions with deliberate self harm being assessed by the oncall psychiatrist. This places a significant demand on psychiatric services and has implications for the patient, for whom a one-off impulsive gesture may result in unnecessary stigmatisation, and whose delay in being discharged from hospital will be increased by the wait for a psychiatric consultation. We have shown that when patients are admitted under o the care of the A\&E department, only one in five is considered to be sufficiently at risk to require psychiatric evaluation on the following day. Forty per cent of cases referred to the oncall psychiatrist required admission to a psychiatric unit. During 199316 patients were admitted directly for psychiatric inpatient treatment from the A\&E department $(0.9 \%$ of all deliberate self harm cases). We feel this is further evidence that the $A \& E$ team can manage patients with deliberate self harm efficiently in an overnight observation ward and successfully identify those at risk of further self harm. We are not aware of any adverse events resulting from the management of our DSH patients but we are following up all such patients admitted to the $A \& E$ observation ward. 
A significant cost saving is achieved by this system of managing DSH patients. Another study has shown that the cost of an inpatient bed for deliberate self harm is in excess of $£ 150$ per day and a psychiatric referral costs almost $£ 100$. Thus by not referring $80 \%$ of admissions under our care to the psychiatrists we were able to make an annual saving of over $£ 45000$. This saving is even greater because of the short time patients spend on the observation ward.

In summary, we feel that only a minority of patients presenting to the $A \& E$ department with deliberate self harm require a psychiatric assessment. What is necessary, however, is an

Appendix

MODIFIED SAD PERSONS SCORE educational system for new members of staff and a departmental protocol which is locally weighted to deal with patients in the A\&E department. ${ }^{7}$ Patients who have suffered significant complications may be admitted under an appropriate specialist. On occasions patients at serious risk may be admitted to a psychiatric ward directly. Others, who in our experience make up one third of all cases, should be admitted to a short stay observation ward where they can be appropriately managed by a specialised, A\&E based, deliberate self harm team and where appropriate follow up can be arranged, if necessary, following discharge.

\begin{tabular}{ll}
\hline & Description \\
\hline $\mathrm{S}=$ Sex & Male \\
$\mathrm{A}=$ Age & $<19$ or $>45$ years \\
$\mathrm{D}=$ Depression or hopelessness & Admits to depression or decreased concentration, appetite, sleep, libido \\
$\mathrm{P}=$ Previous attempts or psychiatric care & Previous inpatient or outpatient psychiatric care \\
$\mathrm{E}=$ Excessive alcohol or drug use & Stigmata of chronic addiction or recent frequent use \\
$\mathrm{R}=$ Rational thinking loss & Organic brain syndrome or psychosis \\
$\mathrm{S}=$ Separated, divorced, or widowed & Well thought out plan or life threatening presentation \\
$\mathrm{O}=$ Organised or serious attempt & No close family, friends, job, or active religious affiliation \\
$\mathrm{N}=$ No social support & Determined to repeat event or ambivalent \\
$\mathrm{S}=$ Stated future intent &
\end{tabular}

1 Joint Sub-Committee of the Staning Medical Advisory Committee. Hospital treatment of acute poisoning. The Hill Report. London: HMSO, 1968

2 Gardner $R$, Hanka $R$, Roberts SJ, Allon-Smith JU, Kings AA, Nicholson R. (1982) Psychological and social accident department. $B M \mathcal{F} 1982 ; 284: 491-3$.

3 Owens D, Dennis M, Jones S, et al. Self poisoning patients discharged from A\&E: risk factors and outcome. $\mathcal{F} R$ Coll Physicians Lond 1991;25:218-22.
4 Hockberger RS, Rothstein RJ. Assessment of suicide potential by non psychiatrists using the SAD PERSONS score. f Emerg Med 1988;6:99-107.

5 Hawton K, Fagg J. Trends in deliberate self poisoning and self injury in Oxford 1976-90. BMF 1992;304:1409-11.

Yeo HM. The cost of treatment of deliberate self-harm. Arch Emerg Med 1992;9:8-14.

7 The general hospital management of adult deliberate self harm - a consensus statement on minimum standards for service provision. Leeds Consensus Conference (November 1992) (in press). 